\title{
Análise radiológica comparativa entre espondilodiscite tuberculosa e inespecífica
}

\author{
Comparative radiological analysis between spondylodiscitis \\ tuberculosis and nonspecific
}

\section{Análisis radiológico comparativo entre espondilolistesis tuberculosa y no específica}

\author{
Bruno Brasil do Couto' \\ Ricardo Shigueaki Galhego Umeta ${ }^{2}$ \\ Maria Fernanda Silber Caffaro ${ }^{3}$ \\ Robert Meves ${ }^{4}$ \\ Elcio Landim ${ }^{5}$ \\ Osmar Avanzi ${ }^{6}$
}

\section{RESUMO}

Objetivo: comparar as imagens radiológicas da espondilodiscite tuberculosa e a inespecífica. Métodos: estudo retrospectivo mediante revisão de prontuários e exames de imagem dos pacientes portadores de discite inespecífica e específica pelo Micobacterium tuberculosis, que realizaram tratamento, pelo Grupo de Coluna do Departamento de Ortopedia e Traumatologia no período de 1989 a Agosto de 2009. Resultados: foram analisados 48 prontuários e arquivos de imagens de pacientes portadores de discite tuberculosa (Tbc) e 31 de pacientes portadores de discite inespecífica. Dos portadores de espondilite tuberculosa, também denominada mal de Pott, 54\% eram do sexo masculino. A idade variou de 3 a 87 anos com média de 44,1 anos. Nos pacientes porta-

\section{ABSTRACT}

Objective: to compare the radiological images of tuberculosis and nonspecific spondylodiscitis. Methods: a retrospective study by reviewing medical records and imaging studies of patients, with nonspecific and specific discitis by Mycobacterium tuberculosis (Pott disease), who underwent treatment at the Spine Group, Department of Orthopedics and Traumatology in the period from 1989 to August 2009. Results: we analyzed 48 medical records and image files of patients with Tbc's discitis and 31 patients with non-specific discitis. Of the patients with Pott disease, $54 \%$ were male, and the age ranged from 3 to 87 with a mean of 44.1 years. In patients with non-specific discitis, $68 \%$ were

\section{RESUMEN}

Objetivo: comparar las imágenes radiológicas de la espondilodiscitis tuberculosis y la no especifica. Métodos: estudio retrospectivo mediante la revisión de registros médicos y los estudios radiológicos de los pacientes con discitis inespecifica y especifica por Mycobacterium tuberculosis (mal de Pott), que se sometieron a tratamiento, en el Grupo de la Columna Vertebral del Departamento de Ortopedia y Traumatología en el período de 1989 a agosto de 2009. Resultados: se analizaron 48 historias clínicas $y$ archivos de imagen de los pacientes con discitis tuberculosis (Tbc) y 31 pacientes con discitis no especifica. De los pacientes con mal de Pott, el $54 \%$ eran del sexo masculino. La edad osciló entre 3 a 87 años con una media de 44,1 años. En los pacientes con discitis no especifi-

\footnotetext{
Trabalho realizado no Departamento de Ortopedia e Traumatologia da Faculdade de Ciências Médicas da Santa Casa de São Paulo - FCMSCSP - São Paulo (SP), Brasil.

'Aluno do curso de aperfeiçoamento do Grupo da Coluna do Departamento de Ortopedia e Traumatologia da Faculdade de Ciências Médicas da Santa Casa de São Paulo - FCMSCSP - São Paulo (SP), Brasil.

${ }^{2}$ Médico Pós-graduando do Departamento de Ortopedia e Traumatologia da Faculdade de Ciências Médicas da Santa Casa de São Paulo - FCMSCSP - São Paulo (SP), Brasil.

${ }^{3}$ Mestre; Professora Instrutora; Assistente do Grupo de Coluna do Departamento de Ortopedia e Traumatologia da Faculdade de Ciências Médicas da Santa Casa de São Paulo - FCMSCSP - São Paulo (SP), Brasil.

${ }^{4}$ Doutor; Professor Assistente e Chefe do Grupo de Coluna do Departamento de Ortopedia e Traumatologia da Faculdade de Ciências Médicas da Santa Casa de São Paulo - FCMSCSP - São Paulo (SP), Brasil.

5Doutor; Professor e Consultor do Grupo de Coluna do Departamento de Ortopedia e Traumatologia da Faculdade de Ciências Médicas da Santa Casa de São Paulo - FCMSCSP - São Paulo (SP), Brasil.

•Doutor; Professor Adjunto do Departamento de Ortopedia e Traumatologia da Faculdade de Ciências Médicas da Santa Casa de São Paulo - FCMSCSP - São Paulo (SP), Brasil.
} 
dores de discite inespecífica, $68 \%$ eram do sexo masculino, e a idade variou de 2 meses a 76 anos. $O$ padrão radiográfico predominante na discite por Tbc foi o acunhamento da região anterior do corpo vertebral em $88,2 \%$ dos casos. Nos pacientes com discite inespecífica, a diminuição do espaço discal foi o achado mais prevalente, encontrado em $87 \%$ dos casos. Conclusão: A discite por Tbc apresentou nesta série uma maior prevalência de alterações estruturais importantes na coluna vertebral, como a cifose, e reação paravertebral em relação à discite inespecífica. Enquanto a prevalência de infecção inespecífica foi maior em pacientes pediátricos, a discite por Tbc teve prevalência progressivamente maior com o aumento da idade dos pacientes. male, and the age ranged from 2 months to 76 years. The predominant radiographic pattern on tuberculosis discitis was the wedging of the anterior vertebral body in $88.2 \%$ of the cases. In patients with non-specific discitis, decreased disc space was the most prevalent finding, found in $87 \%$ of the cases. Conclusion: Tbc's discitis has presented more structural changes in the spine, such as kyphosis, and paravertebral reaction in relation to non-specific discitis. Led to fewer and not as severe vertebral abnormalities generally progressed well with only conservative treatment. While the prevalence of non-specific infection was higher in pediatric patients, Tbc's discitis was more prevalent with the increase of the age of patients. ca, el $68 \%$ eran del sexo masculino, la edad osciló entre 2 meses a 76 años. El patrón radiológico predominante en discitis tuberculosis fue el acuñamiento del cuerpo vertebral anterior en $88,2 \%$ de los casos. En los pacientes con discitis no especifica, la disminución de espacio en el disco fue el hallazgo más frecuente, que se encuentra en el $87 \%$ de los casos. Conclusión: la discitis tuberculosis presentó en esta serie un mayor prevalencia de cambios estructurales importantes en la columna vertebral, como la cifosis y la reacción paravertebral en relación a la discitis no especifica. Por lo cuanto, la prevalencia de infección no especifica fue mayor en pacientes pediátricos, la discitis tuberculosis tubo una prevalencia progresivamente mayor con el aumento de la edad de los pacientes.
DESCRITORES: Coluna vertebral; Discite; Tuberculose/ radiografia
KEYWORDS: Spine; Discitis; Tuberculosis/radiography
DESCRIPTORES: Columna

vertebral; Discitis; Tuberculosis/radiografía

\section{INTRODUÇÃO}

Hipócrates foi o primeiro a descrever a infecção na coluna vertebral. Depois, Galeno relatou o desenvolvimento de deformidade na coluna causado pelo processo infeccioso ${ }^{1}$. A espondilodiscite piogênica é a principal causa de infecção na coluna vertebral; porém, a tuberculose é uma doença que permanece frequente em muitas regiões do mundo, especialmente nos países em desenvolvimento, onde as dificuldades com a saúde pública são maiores, apesar das melhorias sanitárias e terapêuticas, acometendo pacientes com baixas defesas imunológicas, tais como imunocomprometidos por agentes quimioterápicos ou pela Síndrome da Imunodeficiência Adquirida ${ }^{1,2}$.

A tuberculose na coluna vertebral, ou mal de Pott, ocorre quando o Mycobacterium tuberculosis da corrente sanguínea e dos vasos linfáticos penetra na porção anterior do corpo vertebral. A diferenciação clínica entre a discite específica, causada pelo Mycobacterium tuberculosis, e a discite piogênica não específica é fundamental, em virtude de a terapêutica dessas infecções ser diferente. Diagnóstico e intervenção precoce são essenciais para a prevenção de déficit neurológico permanente e deformidade vertebral, mais comum na infecção tuberculosa devido ao envolvimento de múltiplos níveis vertebrais ${ }^{3}$.

A infecção piogênica espinhal engloba uma ampla gama de entidades clínicas, incluindo espondilodiscite, osteomielite vertebral e abscesso epidural. A discite piogênica é uma entidade incomum, com uma incidência de
1 em 100 mil a 1 em 250 mil. Estudos sugerem que a incidência dessa infecção está aumentando ${ }^{4}$. Isso, em parte, pode ser devido à maior expectativa de vida para pacientes com doenças crônicas debilitantes, imunossupressão, uso de esteroides, vírus da HIV, uso de drogas endovenosas e cirurgia espinhal prévia. O diagnóstico de infecção piogênica espinhal costuma ser tardio devido a sintomas muitas vezes inespecíficos ${ }^{4,5}$.

A espondilodiscite na infância, ao contrário do adulto, que apresenta um incremento na sua incidência, é doença rara com incidência não conhecida ${ }^{6}$.

O diagnóstico de infecção piogênica na coluna é realizado por meio de características clínicas, laboratoriais, radiográficas e do isolamento microbiano. $\mathrm{O}$ atraso no diagnóstico pode variar de 2 a 12 semanas e, em alguns casos, pode chegar a até 3 meses, e é esse atraso no diagnóstico que pode levar à destruição do corpo vertebral e a eventual déficit neurológico?

O diagnóstico definitivo de infecção na coluna é realizado por meio do isolamento do micro-organismo na hemocultura ou na cultura de material proveniente da biópsia aberta ou fechada da lesão, que demonstra o agente etiológico em menos de $50 \%$ dos $\operatorname{casos}^{7,8}$.

Nos estágios iniciais da discite inespecífica, as radiografias apresentam sensibilidade e especificidade muito baixas. O sinal radiográfico mais precoce é uma perda na definição e irregularidade do platô vertebral, que aparece de duas a oito semanas após o início dos sintomas ${ }^{8}$. Na suspeita diagnóstica de espondilodiscite, com ausência de achados 
radiográficos característicos, a cintilografia óssea é uma opção de exame sensível para infecção, porém, a ressonância magnética $(\mathrm{RM})$ é o exame de imagem mais útil para a investigação de infecção piogênica na coluna, especialmente nos estágios iniciais, quando outras modalidades de exames de imagem ainda são normais, como as radiografias, ou inespecíficos, como a cintilografia. É altamente sensível (96\%) e específico (94\%) e, por ser não invasivo, é o exame de escolha para condução de uma infecção na coluna ${ }^{8,9}$.

O diagnóstico da tuberculose vertebral é difícil e, em geral, estabelecido em estágio avançado pela imagem radiográfica.

O bacilo da tuberculose inicia a destruição do corpo vertebral e se propaga para a vértebra adjacente por meio dos ligamentos. Nos estágios mais avançados pode ocorrer um colapso vertebral progressivo, resultando na formação de deformidade em cifose vertebral. Radiografias podem revelar lesões avançadas com osteólise vertebral e diminuição do espaço discal, sendo tais achados semelhantes em pacientes com espondilodiscite inespecífica. No entanto, a ausência de esclerose reacional pode favorecer a hipótese de tuberculose. A tomografia computadorizada (TC) possibilita visibilizar o acometimento do corpo e disco vertebral e do abscesso paravertebral, enquanto a RM é útil para determinar a invasão para os tecidos moles e canal medular, além da extensão do abscesso para as vértebras adjacentes. Além disso, a TC é de grande valor no diagnóstico da tuberculose vertebral, demonstrando as alterações mais precocemente do que as radiografias, Porém, o melhor exame de imagem para a tuberculose vertebral é a RM, a qual é mais sensível do que as radiografias e mais específica que a TC. A RM possibilita o diagnóstico da tuberculose vertebral de quatro a seis meses antes dos métodos tradicionais, oferecendo os benefícios do diagnóstico e tratamento precoce ${ }^{3,10}$.

Cumpre ressaltar que, na fase inicial, o diagnóstico diferencial entre a infecção inespecífica (discite piogênica) e específica (tuberculose vertebral) pode ser difícil. Devemos nos basear em dados dos exames laboratoriais, aspectos dos exames de imagem disponíveis e evolução do quadro clínico dos pacientes. Em virtude de atraso no diagnóstico, essas infecções podem ter evolução desfavorável e mais prolongada, podendo resultar em sequelas graves ${ }^{10}$.

A possibilidade de diferenciar os dois tipos de espondilodiscite é de relevância clínica por auxiliar na tomada de decisão, em especial na indicação da antibioticoterapia. Por isso, realizamos análise radiológica comparativa entre a espondilodiscite específica por Tbc e a espondilodiscite inespecífica.

\section{MÉTODOS}

Após aprovação do Comitê de Ética em Pesquisa em Seres Humanos da nossa instituição (número 117/09), foi realizado um estudo retrospectivo, mediante revisão dos prontuários do Serviço de Arquivo Médico e Estatístico da Irmandade Santa Casa de São Paulo, dos pacientes portadores de discite inespecífica e específica pelo Micobacterium tuberculosis, que realizaram tratamento, conservador ou cirúrgico, pelo Grupo de Coluna do Departamento de Ortopedia e Traumatologia no período de 1989 a Agosto de 2009. Foram analisados exames de imagem e prontuários dos pacientes que incluíram: radiografias simples nas incidências anteroposterior e de perfil datadas do início do acompanhamento do paciente em nosso serviço. Nos casos disponíveis, foram avaliadas imagens de TC e RM. O aspecto radiográfico do acometimento vertebral foi comparado em relação à localização do acometimento vertebral, à extensão do processo infeccioso, a alterações do espaço discal, ao colapso ou destruição do corpo vertebral e à formação de abscessos de partes moles.

Como critério de inclusão, consideramos a análise dos exames de imagem - radiografias (RX), TC e RM - dos pacientes portadores de espondilodiscite em que houve resolução dos sinais, sintomas e exames laboratoriais após o teste terapêutico com base em achados da história clínica, antecedentes e exames laboratoriais. A conduta do grupo foi a internação dos portadores de espondilodiscite inespecífica para antibioticoterapia endovenosa com cobertura para Staphylococcus aureus e, após melhora clínica e ambulatorial, manutenção da antibioticoterapia via oral até a resolução do quadro clínico. Nos casos de espondilodiscite especifica por mal de Pott, optou se pela imobilização com órtese tipo toraco lombo sacro (TLSO) ou confecção de gesso associado ao esquema tríplice por pelo menos um ano após período de teste terapêutico inicial. Não realizamos biópsia óssea de rotina nesses pacientes. Para os pacientes portadores de discite inespecífica, foram utilizados como critério de inclusão os casos em que os pacientes apresentavam alteração clínica, laboratorial e radiográfica compatível com o diagnóstico.

Nos pacientes com imagem radiológica mostrando deformidade em cunha do corpo vertebral e cifose da região atingida, procuramos mensurar a cifose localizada e segmentar (Figura 1) por meio do método descrito por Cobb ${ }^{11}$.

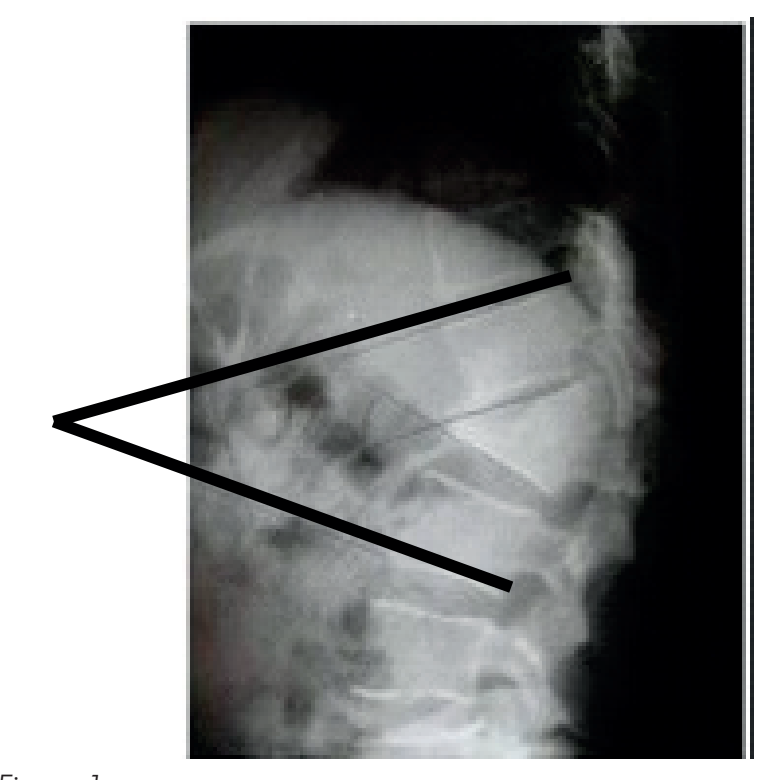

Figura 1

Mensuração do ângulo de cifose segmentar pelo método de Cobb. 
As variáveis utilizadas foram: sexo, idade, nível do acometimento, tempo de doença, imagem compatível no $\mathrm{RX}, \mathrm{TC}$ e RM. A cifose do segmento e do corpo vertebral acometido foi mensurada por meio do ângulo de Cobb.

Para coleta de dados foi aplicado questionário, e o programa Epi Info 2004 será utilizado para a estatística descritiva.

\section{RESULTADOS}

Foram analisados 48 prontuários e arquivos de imagens de pacientes portadores de discite específica ou mal de Pott e 31 prontuários e arquivos de imagens de pacientes portadores de discite inespecífica.

Dos pacientes portadores de mal de Pott, 26 (54\%) eram do sexo masculino e 22 (46\%) do sexo feminino. Em relação à idade, variou de 3 a 87 anos com média de 44,1 anos. Nos pacientes portadores de discite inespecífica, $21(68 \%)$ eram do sexo masculino e 9 (32\%) do feminino (Figuras 2 e 3 ). A idade dos pacientes variou de 2 meses a 76 anos, sendo 8 oito pacientes com idade entre 3 e 10 anos, 3 pacientes com idade entre 10 e 18 anos, nenhum paciente com idade entre 18 e 30 anos, quatro pacientes com idade entre 30 e 50 anos e sete pacientes com mais de 50 anos de idade.

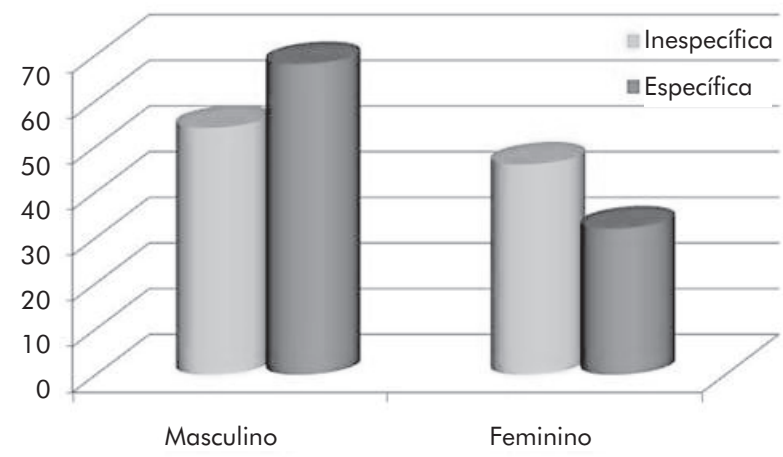

Figura 2

Distribuição por sexo dos pacientes portadores de espondilodiscite específica e inespecífica.

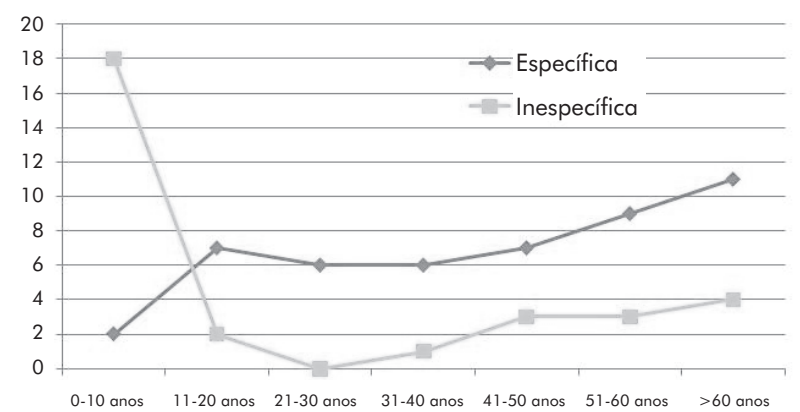

Figura 3

Distribuição por faixa etária dos pacientes portadores de espondilodiscite específica (Tbc) e inespecífica.

Nos pacientes portadores de mal de Pott, foram acometidas as vértebras torácicas $(37,8 \%)$, toracolombares $(6,7 \%)$ e lombares $(55,6 \%)$, sendo que, nos pacientes estudados, não houve acometimento da coluna cervical e lombo-sacro. $\mathrm{O}$ acometimento em uma única vértebra ocorreu em 11,1\% dos pacientes, duas vértebras em $75,6 \% \%$, três vértebras em $8,9 \%$ e cinco vértebras em 4,4\%. Quanto ao nível do acometimento da discite inespecífica, 28 (92,3\%) pacientes apresentaram acometimento de um único nível, e três $(9,7 \%)$ pacientes de mais de um nível. A coluna lombar foi o local de maior acometimento com 28 (92,3\%) casos, seguida pela coluna torácica, em três pacientes. Nenhum paciente apresentou lesão na coluna cervical (Figura 4).

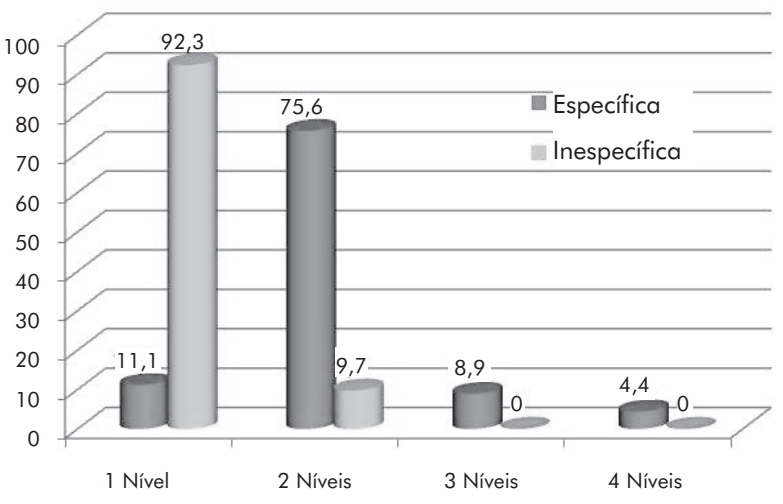

Figura 4

Distribuição quanto ao número de níveis do acometimento nos pacientes com espondilodiscite.

O padrão de imagem (RX, TC e RM) predominante nos pacientes portadores de espondilodiscite específica por mal de Pott foi a deformidade em cunha com redução da altura anterior do corpo vertebral acometido associado à cifose localizada, lesão lítica exuberante na placa e terminal vertebral, pouco ou nenhum comprometimento do disco intervertebral com reação paravertebral, conforme demonstrado nas Figuras 5 e 6 . Abscesso paravertebral foi encontrado em seis pacientes na RM. $\mathrm{O}$ aumento da cifose da região lesada ocorreu em $88,2 \%$ dos pacientes.

Em relação aos pacientes portadores de espondilodiscite inespecífica, imagens radiográficas compatíveis com discite estiveram presentes em 29 dos 31 pacientes. Alterações na RM compatíveis com discite estiveram presentes em 23 pacientes, conforme demonstrado na Figura 7. Em relação à TC, quatro pacientes apresentaram alterações compatíveis com discite. Dos achados radiográficos, a diminuição do espaço discal foi encontrada em 27 pacientes (Figura 8); esclerose e irregularidade dos bordos em 14 pacientes; cifose segmentar de 45 graus em um paciente; sinal de osteomielite vertebral em um paciente; retificação da coluna lombar em um paciente; escoliose antálgica em cinco pacientes (Figura 9); anquilose do nível vertebral acometido em um paciente; e abscesso epidural em um paciente.

\section{DISCUSSÃO}

A espondilodiscite piogênica é a principal causa de infecção na coluna vertebral. Porém, a tuberculose é uma doen- 


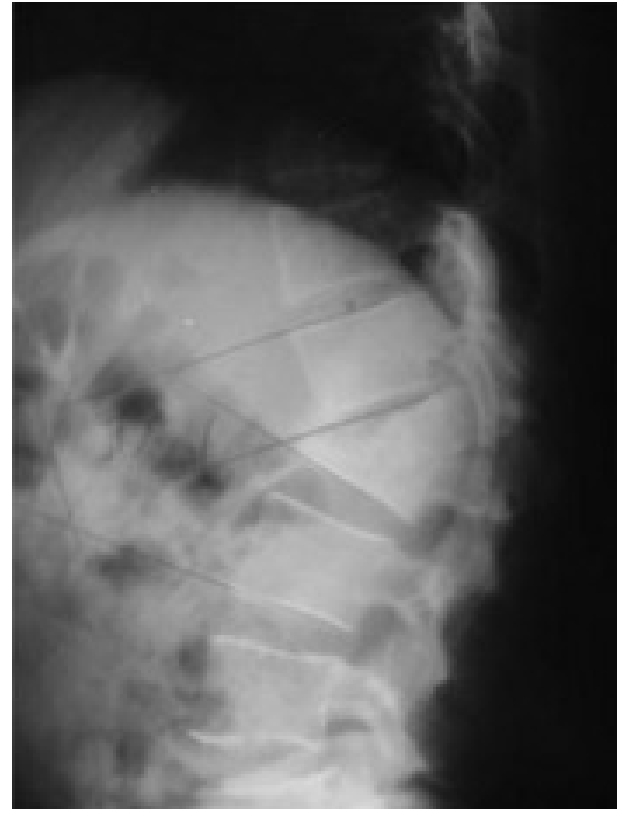

Figura 5

Deformidade cifótica em paciente com espondilodiscite específica.

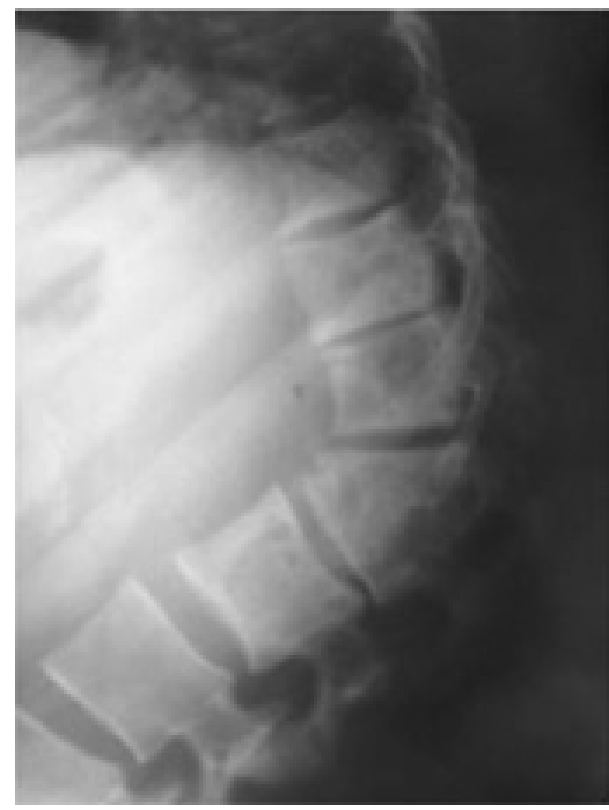

Figura 6

Deformidade cifótica resultante de espondilodiscite específica em outro paciente.

ça que permanece frequente em muitas regiões do mundo, principalmente nos países em desenvolvimento, onde as dificuldades com a saúde pública são maiores, apesar das melhorias sanitárias e terapêuticas, acometendo principalmente pacientes imunocomprometidos ${ }^{1,2}$.

Neste estudo, $68 \%$ dos pacientes que realizaram tratamento de discite eram do sexo masculino. A literatura relata que o sexo masculino é o mais frequentemente acometido pela doença, em um percentual de 56 a $65 \%$. Em relação à faixa etária, este trabalho encontrou 11 pacientes $(35 \%)$ com

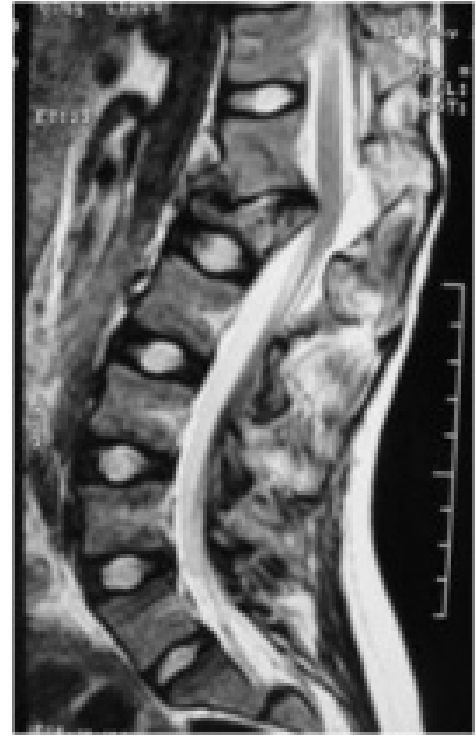

Figura 7

Ressonância magnética de paciente com espondilodiscite tuberculosa.

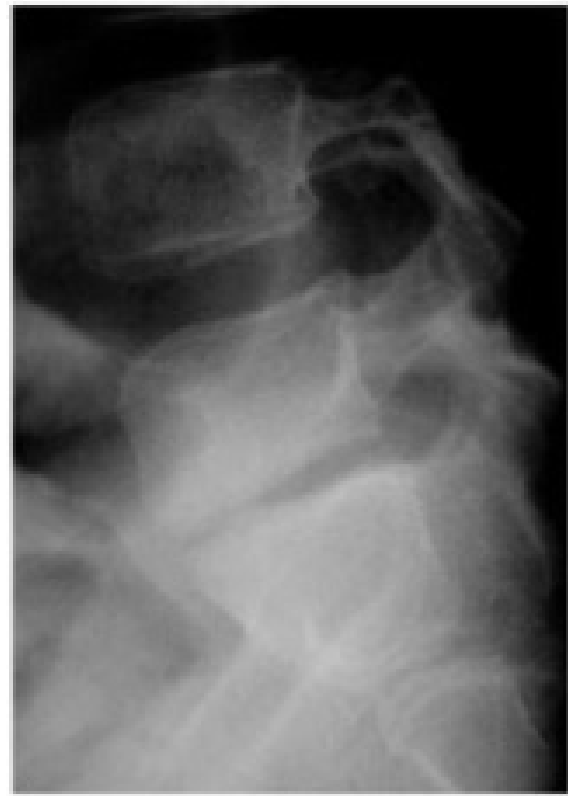

Figura 8

Radiografia de paciente com espondilodiscite inespecífica apresentando diminuição do espaço discal.

menos 18 anos de idade. A literatura coloca a discite como uma doença que acomete principalmente os pacientes com idade acima de 50 anos. Neste trabalho, foram encontrados apenas $22 \%$ dos pacientes nessa faixa etária ${ }^{4}$.

Dos pacientes portadores de mal de Pott, 26 (54\%) eram do sexo masculino e 22 (46\%) do sexo feminino. Em relação à idade, variou de 3 a 87 anos, com média de 44,1 anos. De acordo com a literatura, cerca de $60 \%$ dos pacientes com diagnóstico de tuberculose na coluna vertebral são do sexo feminino. 


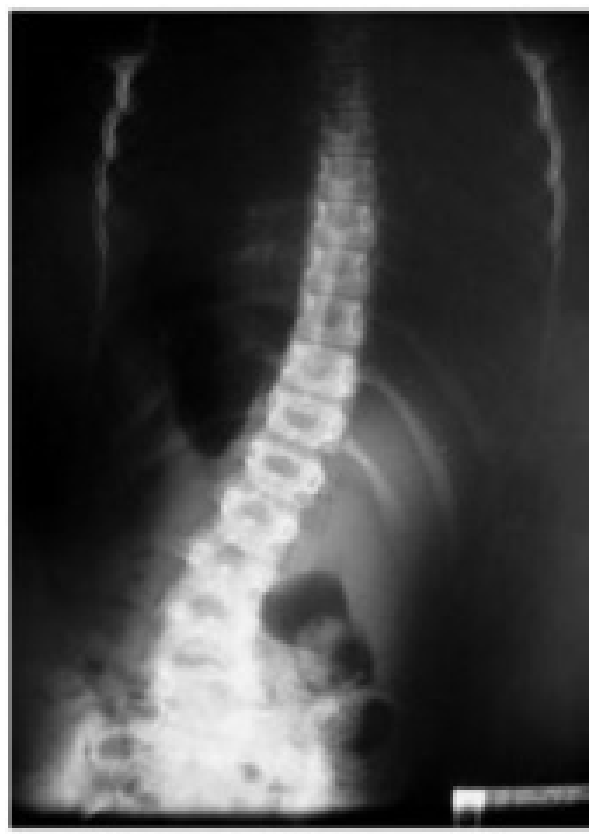

Figura 9

Radiografia de paciente com espondilodiscite inespecífica.

Quanto ao nível de acometimento, 92\% dos pacientes apresentaram acometimento de apenas um nível, e 92\% dos pacientes também apresentaram como região do acometimento a coluna lombar, diferente do que é encontrado na literatura, com cerca de 45 a $60 \%$ dos pacientes com acometimento da região lombar pela discite. Nos pacientes portadores de mal de Pott, o acometimento de um nível ocorreu em seis pacientes, sendo dois torácicos e quatro lombares. Foram acometidos dois níveis em 37 pacientes (15 torácicas e 22 lombares). Em três pacientes, três níveis vertebrais foram acometidos (dois torácicos e um toracolombar), e em dois pacientes, cinco níveis vertebrais foram acometidos, sendo dois toracolombares. De acordo com a literatura, a tuberculose na coluna vertebral apresenta geralmente mais de um nível de acometimento na coluna, sendo a transição toracolombar a região de maior prevalência ${ }^{12}$.
A cifotização da região acometida na coluna vertebral nos pacientes com discite específica ocorreu em $88,2 \%$ dos casos. A literatura relata complicação em cifose da coluna vertebral em cerca de $70 \%$ dos pacientes portadores de tuberculose da coluna vertebral. Já em relação aos pacientes portadores de discite inespecífica, este estudo encontrou complicação em cifose em apenas um paciente. De acordo com a literatura, a alteração radiográfica mais frequente nos pacientes portadores de discite inespecífica é a diminuição do espaço discal, encontrada em $87 \%$ dos pacientes deste estudo ${ }^{10,12}$.

Os aspectos clínicos e laboratoriais constituem importantes fatores na tomada de decisão no momento do teste terapêutico mediante a utilização de antibioticoterapia. Entretanto, os achados indiretos nos exames de imagem discutidos na nossa série, somados ao conhecimento da diferente fisiopatologia entre as duas afecções, são de essencial importância nessa situação clínica, principalmente diante do fato de a biópsia, quando indicada de rotina, apresentar baixa especificidade e sensibilidade. Em síntese, o achado radiológico sugestivo de espondilodiscite inespecífica foi a diminuição do disco intervertebral com irregularidade nas placas terminais vertebrais sem sinais de reação inflamatória paravertebral exuberante. Na específica, foram a cifose localizada com neoformação e a destruição óssea regional associada ao acometimento dos corpos vertebrais, relacionadas à exuberante reação paravertebral.

\section{CONCLUSÃO}

Nesta série, os exames de diagnóstico por imagem mostraram evolução com deformidade cifótica e reação paravertebral mais exuberantes no acometimento da coluna vertebral por tuberculose do que nos casos de espondilodiscite inespecífica. Enquanto a prevalência de infecção inespecífica foi maior em pacientes pediátricos, a infecção disco intervertebral por tuberculose teve prevalência progressivamente maior com o aumento da idade dos pacientes.

\section{REFERÊNCIAS}

1. Butler JS, Shelly MJ, Timlin M, Powderly WG, O’Byrne JM. Nontuberculous pyogenic spinal infection in adults: a 12-year experience from a tertiary referral center. Spine (Phila Pa 1976). 2006;31(23):2695-700.
2. Puertas EB, Chagas JC, Wajchenberg M, D’Orto CC. Avaliação clínica, radiológica e tratamento de 17 pacientes com tuberculose óssea na coluna vertebral. Rev Bras Ortop. 1999;34( 2):113-6.

3. Chang MC, Wu HT, Lee CH, Liu CL, Chen TH. Tuberculous spondylitis and pyogenic spondylitis: comparative magnetic resonance imaging features. Spine (Phila Pa 1976). 2006;31(7):782-8.
4. O'Daly BJ, Morris SF, O'Rourke SK. Long-term functional outcome in pyogenic spinal infection. Spine (Phila Pa 1976). 2008;33(8):E246-53.

5. Genever AV, Douglas DL, Howard AC. Discitis: An important consideration in pyrexia of unknown origins. J Bone Joint Surg Br. 2003;85(Suppl. 2):169-70. 
6. Kayser R, Mahlfeld K, Greulich M, Grasshoff H. Spondylodiscitis in childhood: results of a longterm study. Spine (Phila Pa 1976). 2005;30(3):318-23.

7. An HS, Seldomridge JA. Spinal Infections: diagnostic tests and imaging studies. Clin Orthop Relat Res. 2006;(444):27-33.

8. Avanzi O, Chih LY, Meves R, Mattos C. Tratamento da discite na criança. Rev Assoc Med Bras. 2005;51(2):113-6.
9. Garron E, Viehweger E, Launay F, Guillaume JM, Jouve JL, Bollini G. Nontuberculous spondylodiscitis in children. J Pediatr Orthop. 2002;22(3):321-8.

10.Dass B, Puet TA, Watanakunakorn C. Tuberculosis of the spine (Pott's disease) presenting as 'compression fractures'. Spinal Cord. 2002;40(11):604-8.

11.Cobb JR. Outline for the study of scoliosis. Instr Course Lect. 1948;5:261-75.
12.Teo EL, Peh WC. Imaging of tuberculosis of the spine. Singapore Med J. 2004;45(9):439-44.

\section{Correspondência}

Bruno Brasil do Couto

Rua Dr. Cesário Motta Jr., 112

Vila Buarque - D.O.T, $2^{\circ}$ andar.

CEP 01 227-900 - São Paulo (SP), Brasil.

E-mail: brunobdocouto@yahoo.com.br 